\title{
18. STRATIGRAPHIC RESOLUTION OF LEG 85 DRILL SITES: AN INITIAL ANALYSIS ${ }^{1}$
}

\author{
Nicklas G. Pisias, Oregon State University \\ John A. Barron, U.S. Geological Survey, Menlo Park \\ Catherine A. Nigrini \\ and \\ Dean A. Dunn, University of Southern Mississippi²
}

\begin{abstract}
Biostratigraphic datums determined from the study of radiolarian and diatom microfossils are combined with variations in calcium carbonate content to provide an estimate of stratigraphic resolution for the Leg 85 drill sites. These data sets are used to correlate sites, utilizing techniques which consider the sampling resolution of the primary data. The graphic correlation procedure of Shaw (1964) provides an objective means for estimating the line of correlation between sites. The two microfossil groups are used independently to define two lines of correlation. The inverse mapping technique of Martinson et al. (1982) is used to correlate sites from calcium carbonate data. Comparison of the lines of correlation determined from these three data sets suggests that stratigraphic resolution is on the order of 125,000 to 380,000 yrs. However, these limits of resolution are of the same order as the sampling density for the biostratigraphic and carbonate analyses. This suggests that a higher degree of stratigraphic resolution is possible at these important hydraulically piston-cored sites.
\end{abstract}

\section{INTRODUCTION}

One of the primary objectives of Leg 85 and other hydraulic-piston-coring (HPC) cruises aboard Glomar Challenger was to provide high-quality, continuous sediment sections for "high-resolution" stratigraphic and paleoceanographic studies. The ability to carry out such detailed studies depends in part on the quality of the stratigraphic framework into which sediment sections can be placed.

On Leg 85, five sites were drilled in the eastern and central equatorial Pacific. Coring at four sites, 572, 573, 574 , and 575 , recovered at least $150 \mathrm{~m}$ of sediment using the hydraulic piston corer. By double piston coring most of the upper portion of the sediment column, continuous high-quality sediment sections were recovered. Stratigraphic correlation of these sites is based primarily on biostratigraphic datums. Magnetic stratigraphy is available only in the intervals at Sites 573,574 , and 575 where sediment accumulation rate was lower; thus, it provides only limited stratigraphic resolution (Weinreich and Theyer, this volume). Barron et al. (this volume) have summarized the paleontological and magnetic stratigraphy available for sites drilled during Leg 85 . In this chapter we use a different approach to address the question of stratigraphic resolution. We try to define the precision of correlation attainable for Leg 85 sites and present a strategy for defining high-resolution stratigraphies for marine sediment sections using biostratigraphic and carbonate stratigraphies.

\footnotetext{
${ }^{1}$ Mayer, L., Theyer, F., et al., Init. Repts. DSDP, 85: Washington (U.S. Govt. Printing Office).

2 Addresses: (Pisias) College of Oceanography, Oregon State Univ., Corvallis, OR 97331; (Barron) U.S. Geological Survey, Menlo Park, CA 94205; (Nigrini) 510 Papyrus Drive, La Habra Heights, CA; (Dunn) Department of Geological Science, Univ. So. Miss., Hattiesburg, MS 39406.
}

\section{METHODS}

Our strategy is to correlate all sites in detail, using diatoms and radiolarians, and to correlate using the characteristics of the calcium carbonate records of these pelagic sediment sections. The radiolarian and diatom data are used independently to provide two estimates of the correlation between sites. Correlations based on variations in calcium carbonate content provide a third estimate of stratigraphic correlation between sites. These three stratigraphies enable us to estimate the precision with which sites can be correlated. In all cases, we try to account for the sampling resolution of the biostratigraphic and carbonate data.

\section{BIOSTRATIGRAPHIC CORRELATIONS}

The biostratigraphic data used for correlating sites are the first and last occurrences of numerous radiolarian and diatom species. In general, we have not used datums based on evolutionary transitions or changes in relative-abundance estimates. Such datums are more likely to be related to changes in local oceanographic conditions and may not represent synchronous events. Evolutionary transitions may also reflect differing taxonomic concepts of different investigators. The datums used in this summary, and the depth ranges in which they occur at each site, are listed in Tables 1 and 2. Each of the 91 datums was assigned a number, with the convention that a number with a zero to the right of the decimal place represents a last occurrence and a number with a 1 to the right of the decimal place represents a first occurrence. The depth ranges listed in Tables 1 and 2 were determined from the two samples used to define the event. For a last occurrence, the topmost sample in which the species was found defines the base of the depth range, and the next (overlying) sample (in which the species is absent) defines the top of the depth range.

Detailed stratigraphic correlations based on the biostratigraphic data were made by using the graphic correlation technique developed by Shaw (1964) and recently 
Table 1. Radiolarian datums used in graphic-correlation analysis of Leg 85 sites.

\begin{tabular}{|c|c|c|c|}
\hline $\begin{array}{l}\text { Event } \\
\text { code }^{\mathrm{a}}\end{array}$ & Species & $\begin{array}{l}\text { Lower- } \\
\text { limit } \\
\text { depth } \\
(\mathrm{m})^{\mathrm{b}}\end{array}$ & $\begin{array}{l}\text { Upper- } \\
\text { limit } \\
\text { depth } \\
(\mathrm{m})^{\mathrm{c}}\end{array}$ \\
\hline
\end{tabular}

Site 572

41.1 Buccinosphaera invaginata

43.1 Collosphas universus

44.0 Anthocyrtidium angulare

45.0 Lamprocyrtis neoheteroporus

46.0 Theocorythium vetulum

47.0 Didymocyrtis avita

$48.1^{\mathrm{d}}$ Lamprocyrtis nigriniae

49.1 Theocorythium trachelium

51.1 Anthocyrtidium angulare

52.1 Pterocorys hertwigii

53.1 Pterocanium praetextum

54.1 Lamprocyrtis neoheteroporus

$55.0^{\mathrm{d}}$ Lamprocyrtis heteroporus

$56.0^{\mathrm{e}} \quad$ Stichocorys peregrina

57.0 Phormostichoartus fistula

58.0 Spongaster pentas

59.0 Lychnodictyum audax

60.0 Phormostichoartus doliolum

61.1 Amphirhopalum ypsilon

62.1 Didymocyrtis tetrathalamus

63.1 Spongaster tetras

64.0 Didymocyrtis penultima

65.1 Lamprocyrtis heteroporus

66.0 Solenosphaera omnitubus

$67.1^{\mathrm{d}} \quad$ Didymocyrtis avita

69.0 Spongaster berminghami

$70.1^{\mathrm{d}} \quad$ Spongaster pentas

$71.0^{\mathrm{d}}$ Botryostrobus bramlettei

$72.1^{\mathrm{d}}$ Pterocanium prismatium

$73.1^{\mathrm{d}}$

74.0

$75.0^{\mathrm{d}}$

76.0

77.0

78.0

80.1

80.1
82.1
83.1

83.0

83.0
84.0
85.1

87.1

88.0

88.3

89.0

90.0

$91.1^{\mathrm{d}}$

$93.1^{\mathrm{d}}$

$94.1^{\mathrm{d}}$

95.0

Botryostrobus aquilonaris

Stichocorys delmontensis

Didymocyrtis antepenultima

Siphostichartus corona

Acrobotrys tritubus

Calocycletta caepa

Stichocorys peregrina

Solenosphaera omnitubus

Didymocyrtis penultima

Diartus hughesi

Diartus petterssoni

Acrobotrys tritubus

Diartus hughesi

Stichocorys wolffii

Didymocyrtis antepenultima

Cyrtocapsella cornuta

Dorcadospyris alata

Diartus petterssoni

Cyrtocapsella tetrapera

Didymocyrtis laticonus

Lithopera neotera

Calocycletta virginis

Site 573

\begin{tabular}{llrr}
41.1 & Buccinosphaera invaginata & 1.97 & 0.09 \\
42.0 & Stylatractus universus & 6.31 & 1.97 \\
43.1 & Collosphaera tuberosa & 11.46 & 6.31 \\
44.0 & Anthocyrtidium angulare & 20.76 & 15.81 \\
45.0 & Lamprocyrtis neoheteroporus & 20.76 & 15.81 \\
46.0 & Theocorythium vetulum & 20.76 & 15.81 \\
48.1 & Lamprocyrtis nigriniae & 20.76 & 15.81 \\
49.1 & Theocorythium trachelium & 25.31 & 20.76 \\
50.0 & Pterocanium prismatium & 25.31 & 20.76 \\
51.1 & Anthocyrtidium angulare & 31.32 & 22.0 \\
52.1 & Pterocorys hertwigii & 34.61 & 30.48 \\
53.1 & Pterocanium praetextum & 43.41 & 39.25 \\
54.1 & Lamprocyrtis neoheteroporus & 48.01 & 43.41 \\
$55.0^{\mathrm{d}}$ & Lamprocyrtis heteroporus & 57.09 & 52.10 \\
56.0 & Stichocorys peregrina & 39.25 & 34.61 \\
57.0 & Phormostichoartus fistula & 48.01 & 43.41 \\
\hline
\end{tabular}

Table 1. (Continued).

\begin{tabular}{llll}
\hline & & $\begin{array}{c}\text { Lower- } \\
\text { limit } \\
\text { depth } \\
\text { Event }\end{array}$ & $\begin{array}{c}\text { Upper- } \\
\text { limit } \\
\text { code }\end{array}$ \\
\hline
\end{tabular}

Site 573 (Cont.)

$\begin{array}{llll}58.0 & \text { Spongaster pentas } & 48.01 & 43.4\end{array}$

$\begin{array}{llll}60.0 & \text { Phormostichoartus doliolum } \quad 52.10 \quad 48.01\end{array}$

61.1 Amphirhopalum ypsilon $\quad 57.09 \quad 52.10$

62.1 Didymocyrtis tetrathalamus $\quad 57.09 \quad 52.10$

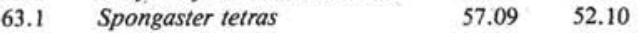

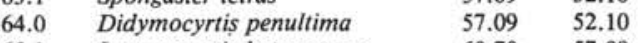

$\begin{array}{llll}65.1 & \text { Lamprocyrtis heteroporus } & 60.70 & 57.09\end{array}$

$\begin{array}{llll}66.0 & \text { Solenosphaera omnitubus } & 76.18 & 70.39\end{array}$

$\begin{array}{lll}67.1^{\mathrm{d}} \quad \text { Didymocyrtis avita } & 76.18 & 70.39\end{array}$

$\begin{array}{llll}69.0 & \text { Spongaster berminghami } \quad 70.39 & 66.60\end{array}$

$\begin{array}{lll}70.1^{\mathrm{d}} \quad \text { Spongaster pentas } & 85.76 & 76.18\end{array}$

$\begin{array}{llll}71.0^{\mathrm{d}} \quad \text { Botryostrobus bramlettei } & 79.99 & 76.18\end{array}$

$72.1 \mathrm{~d} \quad$ Pterocanium prismatium $\quad 70.39 \quad 66.60$

$73.1^{\mathrm{d}} \quad$ Botryostrobus aquilonaris $\quad 85.76 \quad 79.88$

$\begin{array}{llll}74.0^{\mathrm{e}} & \text { Stichocorys delmontensis } & 104.15 & 98.89\end{array}$

$\begin{array}{llll}75.0^{\mathrm{d}} \quad \text { Didymocyrtis antepenultima } & 94.98 & 89.34\end{array}$

$\begin{array}{llll}76.0 & \text { Siphostichartus corona } & 85.76 & 79.88\end{array}$

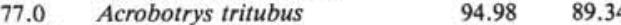

$\begin{array}{llll}78.0^{\mathrm{e}} & \text { Calocycletta caepa } & 94.98 & 89.34\end{array}$

$\begin{array}{llll}80.1 & \text { Stichocorys peregrina } & 122.1 & 122.6\end{array}$

$\begin{array}{llll}81.1 & \text { Solenosphaera omnitubus } & 131.29 & 125.45\end{array}$

82.1 Didymocyrtis penultima $\quad 151.62 \quad 147.01$

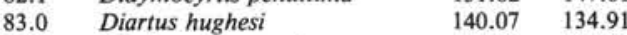

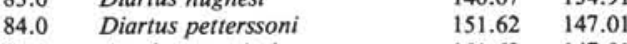

$\begin{array}{llll}85.1 & \text { Acrobotrys tritubus } & 151.62 & 147.01\end{array}$

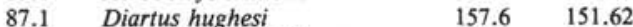

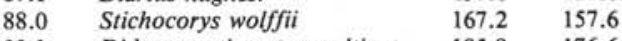

88.3 Didymocyrtis antepenultima $\quad 185.9 \quad 176.6$

$\begin{array}{llll}89.0 & \text { Cyrtocapsella cornuta } & 195.7 & 189.6\end{array}$

$\begin{array}{llll}90.0 & \text { Dorcadospyris alata } & 195.7 & 189.6\end{array}$

$\begin{array}{llll}90.0 & \text { Dorcadospyris alata } & 195.7 & 189.6 \\ 91.1^{\mathrm{d}} & \text { Diartus petterssoni } & 214.7 & 208.6\end{array}$

$92.0^{\mathrm{d}}$ Cyrtocapsella tetrapera $\quad 195.6 \quad 189.6$

$93.1^{d}$ Didymocyrtis laticomus

$94.1^{\mathrm{d}} \quad$ Lithopera neotera $\quad 208.6 \quad 204.3$

$\begin{array}{llll}95.0 & \text { Calocycletta virginis } & 232.8 & 227.6\end{array}$

96.0 Calocycletta costata $\quad 232.8 \quad 227.6$

$\begin{array}{llll}97.1 & \text { Dorcadospyris alata } & 246.6 & 242.8\end{array}$

Site 574

$\begin{array}{llrr}42.0 & \text { Stylatractus universus } & 4.48 & 2.41 \\ 43.1 & \text { Collosphaera tuberosa } & 4.48 & 2.41 \\ 44.0 & \text { Anthocyrtidium angulare } & 5.25 & 4.48 \\ 45.0 & \text { Lamprocyrtis neoheteroporus } & 5.25 & 4.48 \\ 46.0 & \text { Theocorythium vetulum } & 5.25 & 4.48 \\ 48.1^{\mathrm{d}} & \text { Lamprocyrtis nigriniae } & 5.25 & 4.48 \\ 49.1 & \text { Theocorythium trachelium } & 9.75 & 6.75 \\ 50.0 & \text { Pterocanium prismatium } & 8.25 & 6.75 \\ 51.1 & \text { Anthocyrtidium angulare } & 12.75 & 11.25 \\ 52.1 & \text { Pterocorys hertwigii } & 12.75 & 11.25 \\ 53.1 & \text { Pterocanium praetextum } & 12.75 & 11.25 \\ 54.1 & \text { Lamprocyrtis neoheteroporus } & 14.07 & 12.75 \\ 55.0 & \text { Lamprocyrtis heteroporus } & 12.75 & 8.25 \\ 56.0 & \text { Stichocorys peregrina } & 12.75 & 11.25 \\ 57.0 & \text { Phormostichoartus fistula } & 19.20 & 14.07 \\ 58.0 & \text { Spongaster pentas } & 19.20 & 14.07 \\ 60.0 & \text { Phormostichoartus doliolum } & 19.20 & 14.07 \\ 61.1^{\mathrm{e}} & \text { Amphirhopalum ypsilon } & 23.48 & 19.20 \\ 63.1 & \text { Spongaster tetras } & 23.48 & 19.20 \\ 64.0 & \text { Didymocyrtis penultima } & 23.48 & 19.20 \\ 65.1 & \text { Lamprocyrtis heteroporus } & 19.20 & 14.07 \\ 66.0^{\mathrm{e}} \mathrm{d} & \text { Solenosphaera omnitubus } & 23.48 & 19.20 \\ 67.1^{\mathrm{d}} & \text { Didymocyrtis avita } & 23.48 & 19.20 \\ 69.0 & \text { Spongaster berminghami } & 33.10 & 28.73 \\ 70.1^{\mathrm{d}} & \text { Spongaster pentas } & 34.94 & 33.44 \\ 71.0^{\mathrm{d}} & \text { Botroystrobus bramlettei } & 28.73 & 23.48 \\ 72.1^{\mathrm{d}} & \text { Pterocanium prismatium } & 37.94 & 34.94 \\ 77.1^{\mathrm{d}} & \text { Botryostrobus aquilonaris } & 34.94 & 33.44 \\ 74.0 \mathrm{~d} & \text { Stichocorys delmontensis } & 37.94 & 33.10 \\ 75.0^{\mathrm{d}} & \text { Didymocyrtis antepenultima } & 34.94 & 33.10 \\ 76.0 & \text { Siphostichartus corona } & 33.44 & 33.10 \\ 77.0 & \text { Acrobotrys tritubus } & 42.34 & 33.13 \\ & & & \end{array}$


Table 1. (Continued).

\begin{tabular}{|c|c|c|c|}
\hline $\begin{array}{l}\text { Event } \\
\text { code }^{\mathrm{a}}\end{array}$ & Species & $\begin{array}{l}\text { Lower- } \\
\text { limit } \\
\text { depth } \\
\text { (m) b }\end{array}$ & $\begin{array}{l}\text { Upper- } \\
\text { limit } \\
\text { depth } \\
(\mathrm{m})^{\mathrm{C}}\end{array}$ \\
\hline
\end{tabular}

Site 574 (Cont.)

$\begin{array}{llcl}78.0 & \text { Calocycletta caepa } & 51.92 & 42.34 \\ 79.1 & \text { Spongodiscus ambus } & 42.54 & 34.94 \\ 80.1 & \text { Stichocorys peregrina } & 52.0 & 42.5 \\ 81.1 & \text { Solenosphaera omnitubus } & 56.92 & 52.00 \\ 82.1 & \text { Didymocyrtis penultima } & 70.52 & 65.9 \\ 83.0 & \text { Diartus hughesi } & 60.93 & 56.92 \\ 84.0 & \text { Diartus petterssoni } & 70.52 & 65.92 \\ 85.1 & \text { Acrobotrys tritubus } & 65.92 & 60.93 \\ 87.1 & \text { Diartus hughesi } & 75.42 & 70.52 \\ 88.0 & \text { Stichocorys wolffii } & 75.42 & 70.52 \\ 88.3 & \text { Didymocyrtis antepenultima } & 84.92 & 79.87 \\ 89.0 & \text { Cyrtocapsella cornuta } & 108.14 & 103.52 \\ 90.0 & \text { Dorcadospyris alata } & 108.1 & 103.5 \\ 91.1^{\mathrm{d}} & \text { Diartus petterssoni } & 127.1 & 122.7 \\ 92.0^{\mathrm{d}} & \text { Cyrtocapsella tetrapera } & 108.14 & 103.52 \\ 93.1^{\mathrm{d}} & \text { Didymocyrtis laticonus } & 150.9 & 142.2 \\ 94.1^{\mathrm{d}} & \text { Lithopera neotera } & 137.6 & 132.6 \\ 95.0 & \text { Calocycletta virginis } & 157.5 & 152.5 \\ 96.0 & \text { Calocycletta costata } & 162.6 & 157.5 \\ 97.1 & \text { Dorcadospyris alata } & 192.3 & 187.1\end{array}$

Site 575

\begin{tabular}{|c|c|c|c|}
\hline 42.0 & Stylatractus universus & 3.42 & 1.92 \\
\hline 43.1 & Collosphaera tuberosa & 1.92 & 0.42 \\
\hline 46.0 & Theocorythium vetulum & 6.42 & 4.92 \\
\hline $48.1^{\mathrm{d}}$ & Lamprocyrtis nigriniae & 4.92 & 3.42 \\
\hline 49.1 & Theocorythium trachelium & 4.92 & 3.42 \\
\hline 50.0 & Pterocanium prismatium & 4.92 & 3.42 \\
\hline 51.1 & Anthocyrtidium angulare & 6.42 & 4.92 \\
\hline 52.1 & Pterocorys hertwigii & 6.42 & 4.92 \\
\hline 53.1 & Pterocanium praetextum & 6.42 & 4.92 \\
\hline $55.0^{\mathrm{d}}$ & Lamprocyrtis heteroporus & 4.92 & 3.42 \\
\hline 56.0 & Stichocorys peregrina & 6.42 & 4.92 \\
\hline 57.0 & Phormostichoartus fistula & 7.27 & 6.42 \\
\hline 58.0 & Spongaster pentas & 7.30 & 7.27 \\
\hline 59.0 & Lychnodictyum audax & 7.27 & 6.42 \\
\hline 60.0 & Phormostichoartus doliolum & 7.27 & 6.42 \\
\hline 61.1 & Amphirhopalum ypsilon & 7.30 & 7.27 \\
\hline 63.1 & Spongaster tetras & 7.30 & 7.27 \\
\hline 64.0 & Didymocyrtis penultima & 12.9 & 7.3 \\
\hline $66.0^{\mathrm{e}}$ & Solenosphaera omnitubus & 7.27 & 6.42 \\
\hline $69.0 \mathrm{~d}$ & Spongaster berminghami & 12.23 & 9.23 \\
\hline $70.1^{d}$ & Spongaster pentas & 9.23 & 7.73 \\
\hline $71.0^{\mathrm{d}}$ & Botryostrobus bramlettei & 7.73 & 4.92 \\
\hline $72.1^{\mathrm{d}}$ & Pterocanium prismatium & 9.23 & 7.73 \\
\hline $73.1^{d}$ & Botryostrobus aquilonaris & 9.23 & 7.73 \\
\hline 74.0 & Stichocorys delmontensis & 12.23 & 7.27 \\
\hline 76.0 & Siphostichartus corona & 7.27 & 4.92 \\
\hline 77.0 & Acrobotrys tritubus & 10.73 & 6.42 \\
\hline 78.0 & Calocycletta caepa & 15.23 & 12.23 \\
\hline 80.1 & Stichocorys peregrina & 26.3 & 16.8 \\
\hline 81.1 & Solenosphaera omnitubus & 20.23 & 16.89 \\
\hline 82.1 & Didymocyrtis penultima & 26.40 & 20.23 \\
\hline 83.0 & Diartus hughesi & 20.23 & 16.89 \\
\hline 84.0 & Diartus petterssoni & 26.40 & 20.23 \\
\hline 85.1 & Acrobotrys tritubus & 26.40 & 20.23 \\
\hline 87.1 & Diartus hughesi & 31.23 & 26.40 \\
\hline 88.0 & Stichocorys wolffii & 31.23 & 26.40 \\
\hline 88.3 & Didymocyrtis antepenultima & 31.23 & 26.40 \\
\hline 89.0 & Cyrtocapsella cornuta & 44.55 & 38.43 \\
\hline 90.0 & Dorcadospyris alata & 44.5 & 38.4 \\
\hline $91.1^{\mathrm{d}}$ & Diartus petterssoni & 44.55 & 38.43 \\
\hline $92.0^{\mathrm{d}}$ & Cyrtocapsella tetrapera & 44.55 & 38.43 \\
\hline $93.1^{d}$ & Didymocyrtis laticonus & 57.4 & 54.0 \\
\hline $94.1^{\mathrm{d}}$ & Lithopera neotera & 66.9 & 63.5 \\
\hline 95.0 & Calocycletta virginis & 73.0 & $\cdot 66.9$ \\
\hline 96.0 & Calocycletta costata & 76.4 & 73.0 \\
\hline 97.1 & Dorcadospyris alata & 85.1 & 80.2 \\
\hline
\end{tabular}

${ }^{\mathrm{a}}$ Event code: $\mathrm{x} .1=\mathrm{FAD} ; \mathrm{x} .0=\mathrm{LAD}$.

$\mathrm{b}$ Depth of sample used to determine lowest limit of datum.

c Depth of sample used to determine highest limit of datum.

d Datums excluded from graphic correlation of all sites.

e Datums excluded only for individual sites.
Table 2. Diatom datums used in graphic-correlation analysis of Leg 85 sites.

\begin{tabular}{|c|c|c|c|}
\hline $\begin{array}{l}\text { Event } \\
\text { code }^{a}\end{array}$ & Species & $\begin{array}{l}\text { Lower- } \\
\text { limit } \\
\text { depth } \\
(\mathrm{m})^{\mathrm{b}}\end{array}$ & $\begin{array}{l}\text { Upper- } \\
\text { limit } \\
\text { depth } \\
(\mathrm{m})^{\mathrm{c}}\end{array}$ \\
\hline
\end{tabular}

Site 572

$\begin{array}{llrl} & & & \\ 1.0 & \text { Nitzschia reinholdii } & 14.7 & 13.2 \\ 2.0 & \text { Rhizosolenia matuyamaii } & 17.7 & 16.2 \\ 3.1 & \text { Rhizosolenia matuyamaii } & 19.0 & 17.7 \\ 4.0 & \text { Rhizosolenia praebergonii } \text { v. robusta } & 27.2 & 25.7 \\ 5.1 & \text { Pseudoeunotia doliolus } & 33.8 & 32.3 \\ 6.0 & \text { Thalassiosira convexa } & 35.3 & 33.8 \\ 7.0 & \text { Nitzschia jouseae } & 41.5 & 40.0 \\ 8.1 & \text { Rhizosolenia praebergonii } & 49.6 & 48.1 \\ 9.1 & \text { Thalassiosira convexa } \text { v. convexa } & 58.9 & 57.4 \\ 10.1 & \text { Asteromphalus elegans } & 63.4 & 61.9 \\ 11.0 & \text { Nitzschia cylindrica } & 85.5 & 81.8 \\ 12.1 & \text { Nitzschia jouseae } & 100.7 & 95.1 \\ 13.0 & \text { Thalassiothrix miocenica } & 128.3 & 125.7 \\ 14.0 & \text { Asterolampra acutiloba } & 132.0 & 128.3 \\ 15.0 & \text { Nitzschia miocenica } & 140.8 & 136.7 \\ 16.0 & \text { Thalassiosira praeconvexa } & 154.1 & 145.3 \\ 17.1 & \text { Thalassiosira convexa } & 179.5 & 176.7 \\ 18.1 & \text { Thalassiosira praeconvexa } & 198.5 & 192.7 \\ 19.1 & \text { Nitzschia miocenica } & 208.7 & 208.0 \\ 20.0 & \text { Rossiella paleacea } & 208.7 & 208.0 \\ 21.0 & \text { Thalassiosira burckliana } & 219.7 & 217.5 \\ 22.0 & \text { Coscinodiscus yabei } & 227.7 & 227.0 \\ 23.1 & \text { Thalassiosira burckliana } & 257.7 & 255.5 \\ 24.0 & \text { Coscinodiscus vetustissimus } \text { v. javanica } & 260.7 & 257.7 \\ 25.1 & \text { Coscinodiscus vetustissimus } \text { v. } \text { javanica } & 270.2 & 267.2 \\ 26.0 & \text { Asteromphalus moronensis } & 279.7 & 276.7 \\ 27.0 & \text { Coscinodiscus tuberculatus } & 313.2 & 312.5 \\ 28.0 & \text { Denticula punctata } \text { v. hustedtii } & 313.2 & 312.5 \\ 29.0 & \text { Craspedodiscus coscinodiscus } & 322.0 & 316.2 \\ 30.1 & \text { Hemidiscus cuneiformis } & 341.0 & 333.7 \\ 31.1 & \text { Coscinodiscus temperei } \text { v. delicata } & 351.2 & 350.5 \\ 32.0 & \text { Denticula nicobarica } & 369.4 & 363.5 \\ 33.0 & \text { Coscinodiscus lewisianus } & 388.5 & 382.7 \\ 34.1 & \text { Denticula hustedtii } & 408.7 & 407.5 \\ 35.0 & \text { Cestodiscus peplum } & 426.5 & 419.2\end{array}$

Site 573

\begin{tabular}{|c|c|c|c|}
\hline 1.0 & Nitzschia reinholdii & 12.2 & 11.5 \\
\hline 4.0 & Rhizosolenia praebergonii v, robusta & 24.7 & 21.0 \\
\hline 5.1 & Pseudoeunotia doliolus & 30.5 & 24.7 \\
\hline 6.0 & Thalassiosira convexa & 35.7 & 32.7 \\
\hline 7.0 & Nitzschia jouseae & 39.3 & 35.7 \\
\hline 8.1 & Rhizosolenia praebergonit & 46.0 & 43.0 \\
\hline 9.1 & Thalassiosira convexa v. convexa & 51.7 & 48.7 \\
\hline 10.1 & Asteromphalus elegans & 63.7 & 57.7 \\
\hline 11.0 & Nitzschia cylindrica & 66.7 & 63.2 \\
\hline 12.1 & Nitzschia jouseae & 76.2 & 73.4 \\
\hline 13.0 & Thalassiothrix miocenica & 76.9 & 76.2 \\
\hline 14.0 & Asterolampra acutiloba & 85.7 & 76.2 \\
\hline 15.0 & Nitzschia miocenica & 95.2 & 92.4 \\
\hline 16.0 & Thalassiosira praeconvexa & 98.9 & 95.9 \\
\hline 17.1 & Thalassiosira convexa & 112.6 & 110.7 \\
\hline 18.1 & Thalassiosira praeconvexa & 114.6 & 112.6 \\
\hline 19.1 & Nitzschia miocenica & 131.3 & 128.8 \\
\hline 20.0 & Rossiella paleacea & 131.3 & 128.8 \\
\hline 21.0 & Thalassiosira burckliana & 138.0 & 133.5 \\
\hline 22.0 & Coscinodiscus yabei & 140.1 & 142.2 \\
\hline $23.1^{\mathrm{d}}$ & Thalassiosira burckliana & 156.2 & 154.0 \\
\hline 24.0 & Coscinodiscus vetustissimus v. javanica & 167.0 & 158.2 \\
\hline 25.1 & Coscinodiscus vetustissimus v. javanica & 170.7 & 167.0 \\
\hline 26.0 & Asteromphalus moronensis & 170.7 & 167.2 \\
\hline 27.0 & Coscinodiscus tuberculatus & 177.2 & 176.5 \\
\hline 28.0 & Denticula punctata v. hustedtii & 177.2 & 176.5 \\
\hline 29.0 & Craspedodiscus coscinodiscus & 186.7 & 186.0 \\
\hline 30.1 & Hemidiscus cuneiformis & 186.7 & 185.9 \\
\hline 31.1 & Coscinodiscus temperei v. delicata & 199.2 & 195.5 \\
\hline $32.0^{\mathrm{d}}$ & Denticula nicobarica & 210.2 & 205.0 \\
\hline 33.0 & Coscinodiscus lewisianus & 210.2 & 205.0 \\
\hline 34.1 & Denticula hustedtii & 219.7 & 214.5 \\
\hline 35.0 & Cestodisdcus peplum & 224.0 & 222.7 \\
\hline 36.0 & Anaulus californicus & 243.0 & 233.5 \\
\hline 37.1 & Cestodiscus peplum & 254.7 & 252.5 \\
\hline
\end{tabular}


Table 2. (Continued).

\begin{tabular}{|c|c|c|c|}
\hline $\begin{array}{l}\text { Event } \\
\text { code }^{\mathrm{a}}\end{array}$ & Species & $\begin{array}{l}\text { Lower- } \\
\text { limit } \\
\text { depth } \\
(\mathrm{m})^{b}\end{array}$ & $\begin{array}{l}\text { Upper- } \\
\text { limit } \\
\text { depth } \\
(\mathrm{m})^{\mathrm{c}}\end{array}$ \\
\hline
\end{tabular}

\begin{tabular}{llll}
\hline \multicolumn{3}{c}{ Site 573 (Cont.) } \\
38.0 & Thalassiosira fraga & 256.2 & 254.7 \\
39.0 & Thalassiosira bukryi & 267.2 & 265.7 \\
40.1 & Denticula nicobarica & 268.7 & 267.2
\end{tabular}

\section{Site 574}

$\begin{array}{ll}1.0 & \text { Nitzschia reinholdii } \\ 4.0 & \text { Rhizosolenia praebergonii v. robusta } \\ 5.1 & \text { Pseudoeunotia doliolus } \\ 6.0 & \text { Thalassiosira convexa } \\ 7.0 & \text { Nitzschia jouseae } \\ 8.1 & \text { Rhizosolenia praebergonii } \\ 9.1 & \text { Thalassiosira convexa v. convexa } \\ 10.1^{\mathrm{e}} & \text { Asteromphalus elegans } \\ 11.0 & \text { Nitzschia cylindrica } \\ 12.1 & \text { Nitzschia jouseae } \\ 13.0 & \text { Thalassiothrix miocenica } \\ 15.0 & \text { Nitzschia miocenica } \\ 16.0 & \text { Thalassiosira praeconvexa } \\ 17.1 & \text { Thalassiosira convexa } \\ 18.1 & \text { Thalassiosira praeconvexa } \\ 19.1 & \text { Nitzschia miocenica } \\ 20.0 & \text { Rossiella paleacea } \\ 21.0 & \text { Thalassiosira burckliana } \\ 22.0 & \text { Coscinodiscus yabei } \\ 23.1 \mathrm{~d} & \text { Thalassiosira burckliana } \\ 24.0 & \text { Coscinodiscus vetustissimus } \text { v. javanica } \\ 25.1 & \text { Coscinodiscus vetustissimus } \text { v. javanica } \\ 26.0 & \text { Asteromphalus moronensis } \\ 27.0 & \text { Coscinodiscus tuberculatus } \\ 28.0 & \text { Denticula punctata } \text { v. hustedtii } \\ 29.0 & \text { Craspedodiscus coscinodiscus } \\ 30.1 & \text { Hemidiscus cuneiformis } \\ 31.1 & \text { Coscinodiscus temperei } \text { v. delicata } \\ 32.0^{\mathrm{d}} & \text { Denticula nicobarica } \\ 33.0 & \text { Coscinodiscus lewisianus } \\ 34.1 & \text { Denticula hustedtii } \\ 35.0 & \text { Cestodiscus peplum } \\ 36.0 & \text { Anaulus californicus } \\ 37.1 & \text { Cestodiscus peplum } \\ 38.0 & \text { Thalassiosira fraga } \\ 39.0 & \text { Thalassiosira bukryi } \\ 40.1 & \text { Denticula nicobarica } \\ & \\ & \\ & \end{array}$

\begin{tabular}{rr}
6.7 & 4.5 \\
8.2 & 6.7 \\
14.0 & 9.7 \\
14.0 & 9.7 \\
14.0 & 9.7 \\
16.2 & 14.0 \\
23.5 & 19.2 \\
29.7 & 23.5 \\
33.0 & 29.7 \\
33.0 & 29.7 \\
35.2 & 33.0 \\
42.5 & 39.7 \\
44.7 & 42.5 \\
52.0 & 44.7 \\
52.0 & 44.7 \\
57.2 & 54.2 \\
57.2 & 54.2 \\
61.4 & 60.9 \\
64.7 & 61.7 \\
70.5 & 70.1 \\
72.2 & 70.5 \\
80.0 & 75.7 \\
80.0 & 78.7 \\
90.2 & 89.0 \\
91.7 & 90.2 \\
98.6 & 93.2 \\
103.5 & 100.5 \\
113.0 & 110.3 \\
128.7 & 124.5 \\
132.6 & 129.8 \\
144.8 & 142.6 \\
154.7 & 152.5 \\
177.5 & 172.5 \\
197.2 & 194.4 \\
194.2 & 192.3 \\
223.0 & 213.5 \\
226.7 & 224.9 \\
\hline & \\
\hline
\end{tabular}

Site 575

$\begin{array}{ll}1.0 & \text { Nitzschia reinholdii } \\ 4.0 & \text { Rhizosolenia praebergonii v. robusta } \\ 5.1 & \text { Pseudoeunotia doliolus } \\ 6.0 & \text { Thalassiosira convexa } \\ 7.0 & \text { Nitzschia jouseae } \\ 8.1 & \text { Rhizosolenia praebergonii } \\ 12.1 & \text { Nitzschia jouseae } \\ 13.0 & \text { Thalassiothrix miocenica } \\ 15.0 & \text { Nitzschia miocenica } \\ 16.0 & \text { Thalassiosira praeconvexa } \\ 17.1 & \text { Thalassiosira convexa } \\ 19.1 & \text { Nitzschia miocenica } \\ 20.0 & \text { Rossiella paleacea } \\ 21.0 & \text { Thalassiosira burckliana } \\ 22.0 & \text { Coscinodiscus yabei } \\ 23.1 & \text { Thalassiosira burckliana } \\ 26.0 & \text { Asteromphalus moronensis } \\ 27.0 & \text { Coscinodiscus tuberculatus } \\ 28.0 & \text { Denticula punctata } \\ 29.0 & \text { Craspedodiscus coscinodiscus } \\ 30.1 & \text { Hemidiscus cuneiformis } \\ 31.1 & \text { Coscinodiscus temperei v. delicata } \\ 32.0 & \text { Denticula nicobarica } \\ 33.0 & \text { Coscinodiscus lewisianus } \\ 34.1 & \text { Denticula hustedtii } \\ 35.0 & \text { Cestodiscus peplum } \\ 36.0 & \text { Anaulus californicus }\end{array}$

$\begin{array}{rr}3.7 & 0.7 \\ 5.2 & 3.7 \\ 5.2 & 3.7 \\ 5.2 & 3.7 \\ 6.5 & 5.0 \\ 7.3 & 6.7 \\ 7.8 & 7.3 \\ 7.8 & 7.3 \\ 12.5 & 11.0 \\ 12.5 & 11.0 \\ 15.3 & 13.8 \\ 20.5 & 19.0 \\ 20.5 & 19.0 \\ 25.0 & 23.5 \\ 25.0 & 23.5 \\ 27.0 & 26.3 \\ 28.5 & 27.0 \\ 31.5 & 28.5 \\ 35.0 & 34.5 \\ 34.3 & 32.8 \\ 39.9 & 36.9 \\ 44.5 & 40.2 \\ 49.7 & 46.7 \\ 54.0 & 49.7 \\ 63.5 & 56.2 \\ 73.0 & 65.7 \\ 80.2 & 75.2\end{array}$

Table 2. (Continued).

\begin{tabular}{|c|c|c|c|}
\hline $\begin{array}{l}\text { Event } \\
\text { code }^{\mathrm{a}}\end{array}$ & Species & $\begin{array}{l}\text { Lower- } \\
\text { limit } \\
\text { depth } \\
(\mathrm{m})^{b}\end{array}$ & $\begin{array}{l}\text { Upper- } \\
\text { limit } \\
\text { depth } \\
(\mathrm{m})^{\mathrm{c}}\end{array}$ \\
\hline \multicolumn{4}{|c|}{ Site 575 (Cont.) } \\
\hline 37.1 & Cestodiscus peplum & 98.6 & 91.3 \\
\hline 38.0 & Thalassiosira fraga & 98.6 & 91.3 \\
\hline $39.0^{\mathrm{d}}$ & Thalassiosira bukryi & 107.4 & 105.9 \\
\hline 40.1 & Denticula nicobarica & 123.8 & 123.3 \\
\hline 44.0 & Coscinodiscus oligocenicus & 179.2 & 177.9 \\
\hline 45.0 & Thalassiosira primalabiata & 197.9 & 197.3 \\
\hline
\end{tabular}

${ }^{a}$ Event code: $x .1=$ FAD; $x .0=$ LAD.

b Depth of sample used to determine lowest limit of datum.

c Depth of sample used to determine highest limit of datum.

d Datums excluded from graphic correlation of all sites.

e Datums excluded for individual sites.

modified and applied to deep-sea sediments (Pisias et al., 1984). In this technique, all cores are compared to a "standard" section. We have used the section at Site 573 as the standard because of its length and relatively high sediment accumulation rates. A Miocene/Pliocene hiatus or interval of low accumulation rate at about $80 \mathrm{~m}$ sub-bottom, and the possible short hiatuses in the upper and middle Miocene (Barron et al., this volume) at this site, do, however, limit the usefulness of this site in parts of the Miocene.

We have assumed that the biostratigraphic events listed in Tables 1 and 2 represent synchronous events, so that the graphic correlation plots can be used to determine relative changes in sedimentation and the presence of sedimentation anomalies. In Figures 1 and 2, the depth ranges of the diatom and radiolarian datums are plotted for Sites 572, 574, and 575 versus the depth ranges at Site 573. Ideally, if all datums are synchronous and the first or last occurrence of a species in a sediment section represents its true evolutionary appearance or extinction, then the "line" of correlation between any site and the standard site would pass through all correlation boxes. As seen in Figures 1 and 2, the correlation "line" between any one site and Site 573 can be described by a few line segments. Line segments were drawn by hand so that as few segments as possible were used to connect the largest number of correlation boxes.

Several explanations are possible for an event that does not fall on a correlation "line." A last appearance of a species may occur too soon or a first appearance may be delayed because of local environmental conditions. The original graphic correlation method developed by Shaw (1964) assumes that any one site does not necessarily contain the true first or last occurrence, and that by correlating many sites into the standard section the "true" relative position of an event will be defined. In this analysis we deviate from Shaw's (1964) original approach by assuming that, for the most part, the biostratigraphic events represented in each core are the true first or last appearances of a given species. There are several events for which this cannot be so, and these are eliminated from the detailed correlation analysis.

As described by Shaw (1964), changes in the slopes of these line segments represent changes in relative sedi- 
ment accumulation rates between sites, and any offsets in the lines reflect a hiatus in one section and/or expanded accumulation rates in the other. By comparing many sections to the standard, it is possible to determine which is the likely cause of any one observed offset.

In Figures 1 and 2, a number of offsets, some common to both microfossil groups, can be seen. The diatom graphic correlation plot for Site 572 (Fig. 2A) shows a marked offset at about $80 \mathrm{~m}$ sub-bottom at Site 573 , suggesting a hiatus at Site 573 , which is represented by at least $30 \mathrm{~m}$ of section at Site 572 . The radiolarian datums do not clearly show this hiatus, although several datums in this depth interval apparently represent nonsynchronous behavior in the datums. An offset at about $60 \mathrm{~m}$ sub-bottom at Site 572 is seen in both the radiolarian and diatom plots (Figs. 1A and 2A). This offset suggests a short hiatus at Site 572 , or a slightly expanded section at Site 573 at about $70 \mathrm{~m}$ sub-bottom. An expanded section at Site 573 is not inconsistent with the Site 574 correlation plots (Figs. 1B and 2B). Thus, it is possible that a hiatus is present at both Site 574 and Site 572 in this interval or that Site 573 has a slightly expanded section at about $70 \mathrm{~m}$ sub-bottom. Unfortunately, the very low sediment accumulation rates or hiatuses at Site 575 in this interval do not allow the use of this site to clarify the cause of the offset in Figures 1A and 2A.

A similar situation is observed for the offset in the graphic correlation plot for Site 572 at about $35 \mathrm{~m}$ subbottom at that site. The diatom correlation shows a possible hiatus at Site 572 or an expanded section at Site 573 (Fig. 2A). Inspection of the Site 574 plot (Fig. 2B) shows a similar compression at Site 574 as compared with Site 573. The radiolarian data do not indicate an offset at $35 \mathrm{~m}$ sub-bottom at Site 572 (Fig. 1A), but do show either a compressed section at Site 574 or an expansion at Site 573 in this same interval (Figs. 1B and $2 \mathrm{~B} ; 35$ to $40 \mathrm{~m}$ sub-bottom at Site 573 ). Without other sites for comparison, the simplest assumption is that Site 573 has a slightly higher sediment accumulation rate and expanded section in this interval, and that Sites 572 and $\mathbf{5 7 4}$ have complete sections. It is possible that the diatom datum, the first appearance of Pseudoeunotia doliolus at Site 572, is diachronous (Barron et al., this volume).

The correlation plots for Site 575 suggest that the interval of low accumulation rate noted in the upper section at this site (site chapter, this volume), is made up of several short hiatuses separated by intervals of low sediment accumulation rates.

Finally, it is important to note that the major offset and apparent hiatus at Site 573 at $80 \mathrm{~m}$ sub-bottom is not evident in the Site 574 plots (Figs. 1B and 2B). Thus, if this is a hiatus at Site 573 , then it must be present at Site 574 as either a hiatus or a period of greatly reduced accumulation rate. For further discussion of possible hiatuses at Leg 85 sites, see Barron et al. (this volume).

To refine the correlation between all sites and the standard section (Site 573), we utilized Prell's (pers. comm.) modification of the graphic correlation technique, as applied to late Pleistocene isotopic records by Pisias et al.
(1984). From each correlation plot and set of correlation lines, the depth ranges of all datum levels defined at Sites 572,574 , and 575 can be expressed in terms of depth at Site 573. These depth ranges were determined from leastsquares regression lines calculated from the event "boxes" contained within the interval spanned by the line segments drawn in the graphic correlation plots (Figs. 1 and 2).

The depth intervals calculated for the biostratigraphic events were then plotted as in Figure 3 to determine the interval common to all the sites. The common depth interval is considered to be the "best" estimate of where the biodatum should occur within the standard section. Not all events have overlapping depth intervals when projected onto the standard section. This may reflect nonsynchronous behavior of a stratigraphic datum, or the inadequacy of the correlation "line" for an individual site. Datums that clearly cannot represent synchronous events at any one site (datums that predict unrealistic relative accumulation-rate patterns, such as negative slopes in the line of correlation) were removed from further calculations. In some cases, new line segments were drawn and the projected depth intervals of all events were recalculated. The events that had overlapping intervals are indicated in Tables 1 and 2.

Given the depth interval common to all sites, the midpoint is used as the estimate of the true position of the biostratigraphic event in the standard section. The leastsquares regression lines used to define each line of correlation between Site 573 and all other sites allow this depth estimate to be expressed in terms of the sub-bottom depth at Sites 572, 573, and 575. Thus, the diatom and radiolarian datums produce independent correlation lines for all sites with Site 573 (Fig. 4). Note that by using graphic correlation, we have incorporated the resolution with which datums were determined in the correlation estimates.

\section{CARBONATE CORRELATIONS}

The carbonate data used in this summary are from the carbonate-bomb analyses done on board ship for percent $\mathrm{CaCO}_{3}$. The results of the high-resolution studies of Sites 572 and 574 (Pisias and Shackleton, this volume; Pisias and Prell, this volume) suggest that the shipboard data have some limitations in resolving part of the true variability in calcium carbonate deposition at the equatorial Pacific sites, but many similar features in the carbonate signatures of these sites can be resolved by the low-resolution shipboard data and used for stratigraphic purposes.

To provide an objective means of correlation, the inverse mapping technique described by Martinson et al. (1982) was used to correlate Sites 572, 574, and 575 with the standard section at Site 573. This technique utilizes an inverse procedure to find a line of correlation (referred to as a mapping function) which maximizes the correlation coefficient between the two records being correlated. The mapping function is parameterized by a series of weighted sine functions, and the weights are calculated to maximize the linear correlation coefficient between the carbonate records being compared. Thus, the 
A Site 573 sub-bottom depth $(\mathrm{m})$

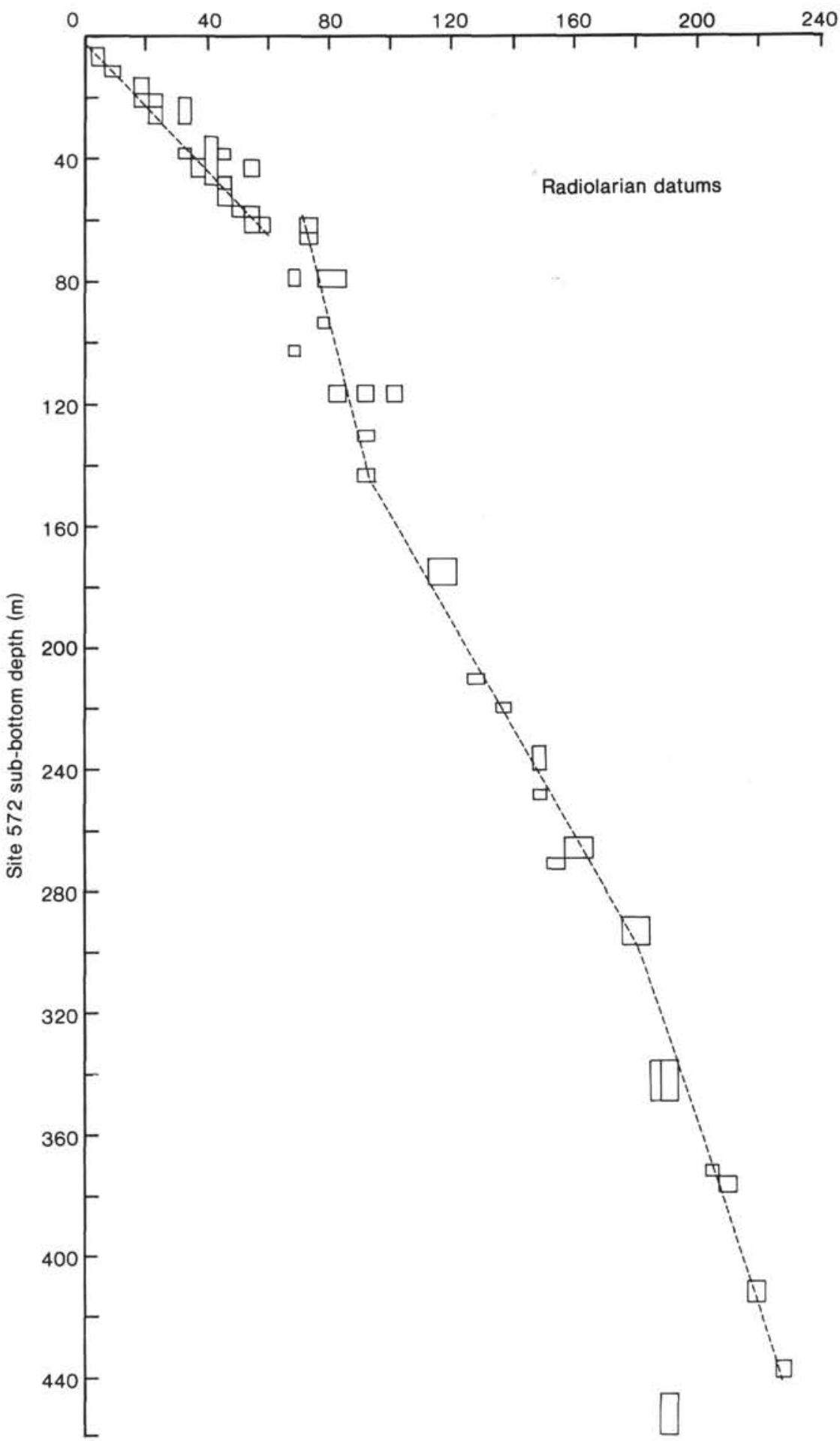

Figure 1. Shaw plots showing correlation of Leg 85 sites based on radiolarian datums given in Table 1. A. Site 572 versus Site 573. B. Site 574 versus Site 573. C. Site 575 versus Site 573.

carbonate records are aligned on the basis of their common characteristics, with regard to both the number of events in an interval and the relative amplitudes of these events. Because the technique has the potential of converging to "local" maxima in the correlation coefficient
(Martinson et al., 1982), and because of the large differences in sediment accumulation rates observed for the Leg 85 sites, the inverse-mapping calculations were made using the biostratigraphic data as initial guesses for the true mapping function. Thus, the estimated line of cor- 
B Site 573 sub-bottom depth (m)

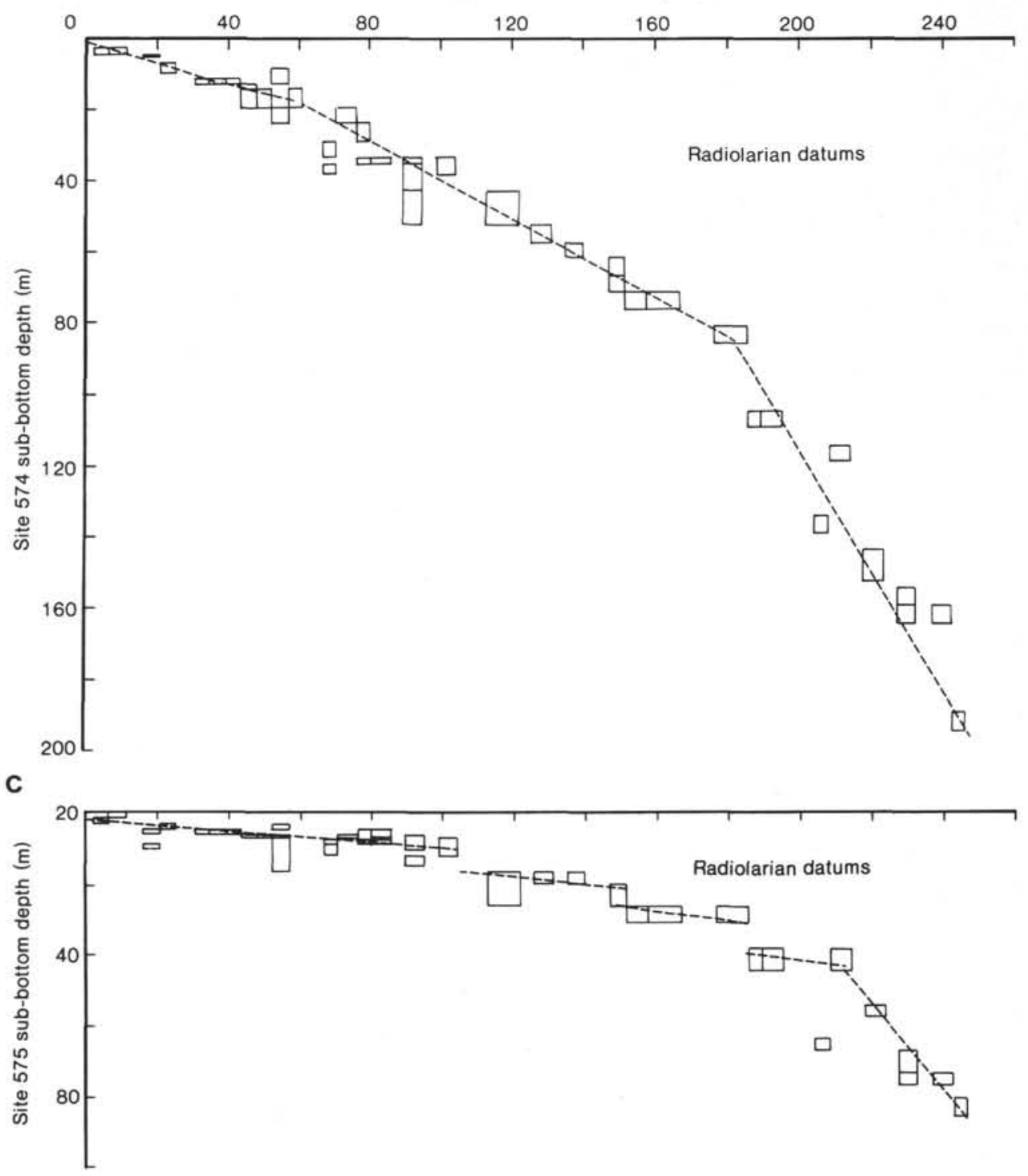

Figure 1. (Continued).

relation from the carbonate data is not independent of the biostratigraphic data.

The inverse-mapping calculations were made twice, each time a different fossil group provided the initial correlation. The results of correlating Sites 574 and 573, using the radiolarian data as an initial guess, are shown in Figure 5. The final correlation coefficients obtained between the Site 573 carbonate record and Sites 572, 574 , and 575 were $0.21,0.75$, and 0.65 , respectively. Except for Site 572, the correlation maps calculated from the two different starting guesses were essentially identical. Because of this convergence in the inverse calculations, we assume that the carbonate correlation provides a third estimate for the true lines of correlation between Sites 573, 574, and 575. Because of the low total correlation and the lack of convergence for the Site 572 data, we do not use the carbonate correlation for this site to estimate the precision of correlation for Site 572.

\section{RESULTS AND DISCUSSION}

The results of both the biostratigraphic and carbonate correlations for Sites 572, 574, and 575 are shown in Figure 4 . We have used these plots to estimate the precision of correlation at each site. These estimates were made by tabulating the ranges (in meters at Site 573) defined by the diatom, radiolarian, and carbonate lines of correlation. Estimates were made at each level where a diatom or radiolarian event occurred at both sites. For Site 572 these ranges are defined from the biostratigraphic data only. These intervals are represented as horizontal lines in Figure 3. The general statistics of these intervals of resolution are given in Table 3. The data presented in Table 3 suggest that the resolution of correlation for these sites is on the order of $4 \mathrm{~m}$ depth at Site 573 . For example, a given depth at Site 574 can be correlated with a depth interval at Site 573 within a mean error of 
N. G. PISIAS, J. A. BARRON, C. A. NIGRINI, D. A. DUNN

A

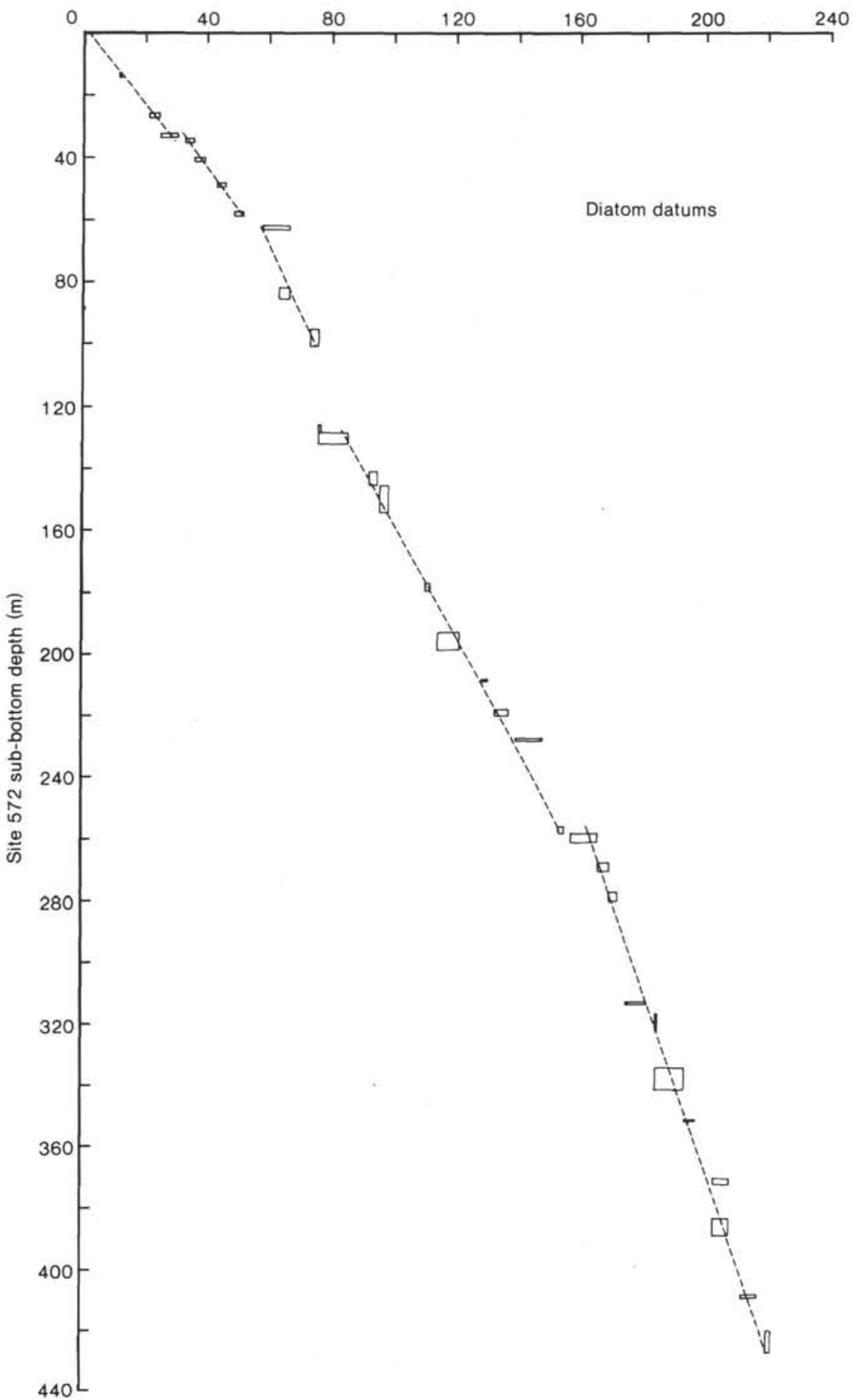

Figure 2. Shaw plots showing correlation of Leg 85 sites based on diatom datums given in Table 2 .

A. Site 572 versus Site 573. B. Site 574 versus Site 573. C. Site 575 versus Site 573 .

$\pm 3.8 \mathrm{~m}$ (Table 3). Given the mean sediment accumulation rates at these sites, this indicates that a stratigraphic resolution on the order of 125,000 to 380,000 yrs. is possible. The error of correlation for Site 575 is larger than for the other sites. If, however, we exclude the interval of lowered accumulation rates or possible hiatuses, the mean stratigraphic error is also close to $4 \mathrm{~m}$.
There are two primary limiting factors to the stratigraphic resolution based on biostratigraphic datums: (1) the number of datums within the interval being correlated and (2) the precision with which the stratigraphic datums are defined.

The estimated resolution of 125,000 to 380,000 yrs. (based on the datums considered in this study and the 


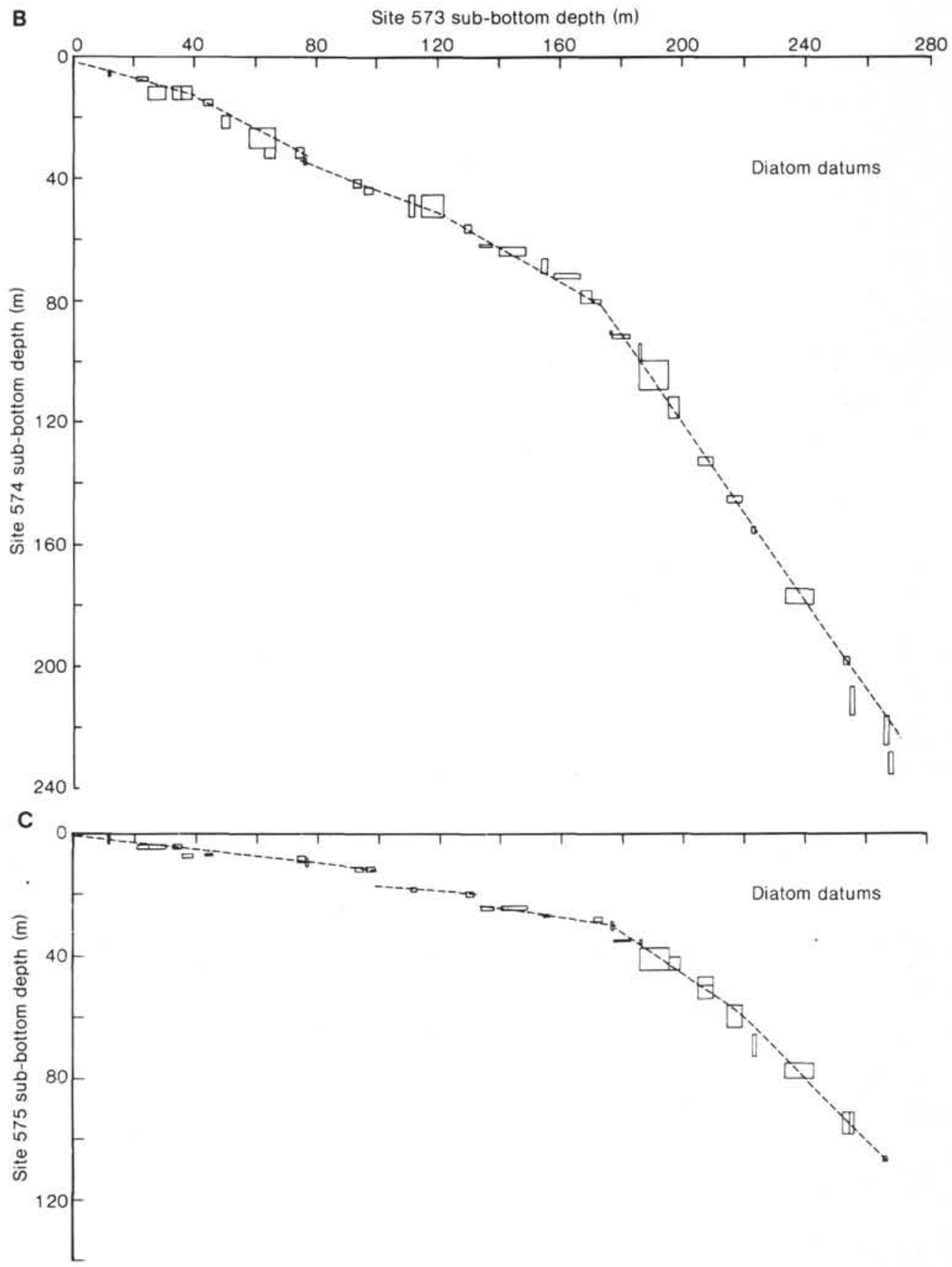

Figure 2. (Continued).

sampling intervals) is not unlike estimated stratigraphic resolution based on studies of isotopic records (Loutit et al., 1983) and that based on analysis of the distribution and number of biodatums in the Neogene (Moore and Romine, 1981). This range of correlation is not satisfactory, however, for the type of high-resolution studies that can be completed on HPC material. It is important, though, to consider the resolution of the data used for stratigraphy. Table 4 summarizes the resolution of the biodatums for each site. Note that the range to which datums are determined is very similar to the estimated stratigraphic resolution. Also, the mean sample interval of the carbonate data from Site 573 limits the resolution of correlation based on carbonate records to about $3.8 \mathrm{~m}$ (twice the mean sampling interval).
Thus, the precision to which the biodatums are determined appears to be an important factor limiting the precision of correlation, as might be expected. This is encouraging, given the lack of detailed magnetic stratigraphy for the Leg 85 drill sites. The results suggest that problems associated with biostratigraphic markers may not severely limit our ability to correlate these equatorial Pacific sites in detail and that it may be possible, with more precise biostratigraphic studies, to determine a highresolution stratigraphic framework for Leg 85 sites.

To provide an estimate of stratigraphic resolution, we have used the biostratigraphic and carbonate data independently. Theoretically, combining the data should increase the possible stratigraphic resolution (assuming that all datums are reliable and that unique features of the 


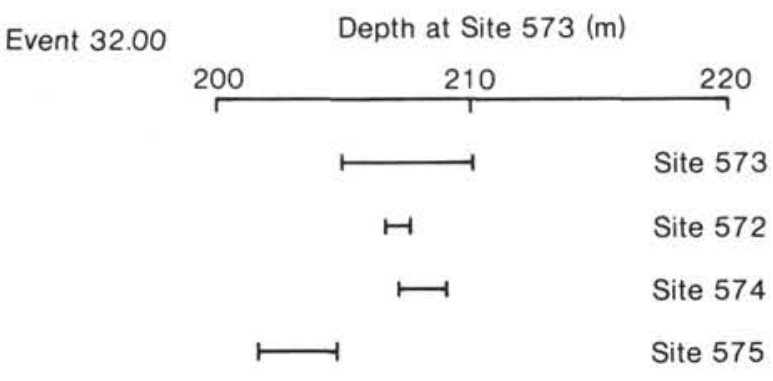

Event 33.00

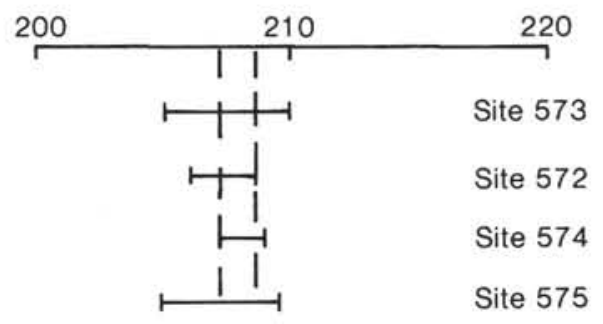

Event 34.10

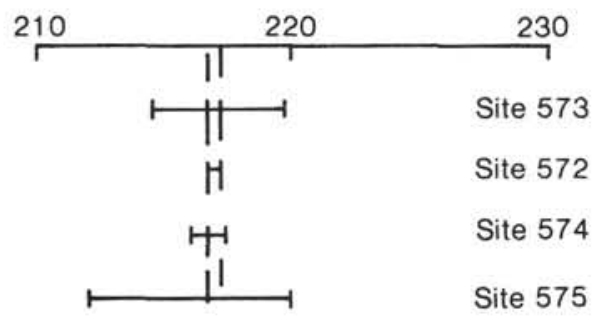

Event 35.00

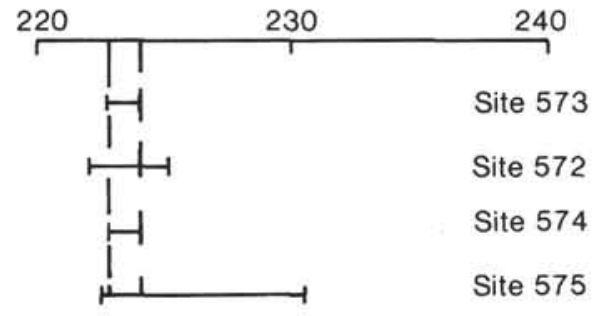

Event 36.00

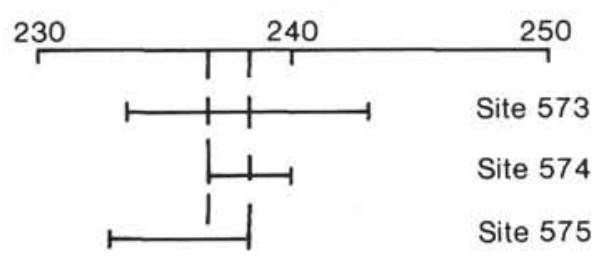

Figure 3. Example of overlapping ranges of datums projected into Site 573.

carbonate variations can be identified). For example, radiolarian and diatom biostratigraphies provide over 91 datums in the $250 \mathrm{~m}$ from Site 573 examined in this analysis. If these datums were uniformly distributed, then correlation to within $2.5 \mathrm{~m}$ depth at Site 573 should be possible. As already noted above, however, this is a finer scale than the sampling resolution for this site.

One of the initial assumptions of the study was that the biostratigraphic datums determined for the Leg 85 sites represented time-correlative events. The graphic cor- relation analysis used here provides a means to evaluate datums that may not satisfy this assumption. Tables 1 and 2 indicate species that did not form overlapping intervals within the graphic correlation analysis. Inconsistent stratigraphic occurrences were noted for several of these species (Nigrini, this volume; Barron, this volume). Some of these datums are based on species that proved to be rare at some of the Leg 85 sites (radiolarians: Spongaster pentas, Pterocanium prismatium [at the lower end of its range]; diatoms: Thalassiosira burckliana), which makes determination of their first or last occurrences difficult. Diatom species Asteromphalus elegans and Denticulopsis nicobarica are relatively fragile, so the dissolution of silica may affect their datums.

Though we have not used datums based on evolutionary transitions, several radiolarian events are based on species that make up well-known lineages. A number of these datum levels proved to be inconsistent. Didymocyrtis penultima, D. avita, and the lineage Lamprocyrtis heteroporus and $L$. neoheteroporus all showed inconsistent behavior (note that Nigrini, this volume, excluded the ancestors of $L$. nigriniae from her analysis because these species were very rare in tropical sediments). Detailed analysis of the Lamprocyrtis group at Site 573 shows that the occurrence of some of these species is sporadic, and there seem to be some inconsistencies in defining the distinguishing characteristics of $L$. heteroporus and $L$. neoheteroporus. It is possible that some of the inconsistencies in datums result from hiatuses at some of the Leg 85 sites.

\section{CONCLUSION}

Because of time limitations and the amount of new biostratigraphic data being generated from material collected during Leg 85 , this summary does not contain all datums determined from the study of radiolarians (Nigrini, this volume) and diatoms (Barron, this volume) or all datums based on calcareous microfossils. Also, we have not fully used the graphic stratigraphy of Shaw (1964) to develop a standard section for the equatorial Pacific. To develop an estimate of stratigraphic resolution, this step was not necessary. Before developing a standard, it would be important to include data from the many other equatorial sites. Finally, it it likely that Site 573 is not the "best" site to use as a standard for Leg 85 ; but to make as objective an estimate of resolution as possible, we chose to use a single site rather than a composite section from a number of sites. This initial analysis of stratigraphic resolution does strongly suggest, however, that sampling resolution is the primary limiting factor in controlling the degree to which the HPC sites in the equatorial Pacific can be correlated.

\section{ACKNOWLEDGMENTS}

This research was supported in part by National Science Foundation Grant OCE83-15471, through the Submarine Geology and Geophysics Section of Ocean Sciences.

\section{REFERENCES}

Loutit, T. S., Pisias, N. G., and Kennett, J. P., 1983. Pacific Miocene carbon isotope stratigraphy using benthic foraminifera. Earth Planet. Sci. Lett., 66:48-62. 
Martinson, D. G., Menke, W., and Stoffa, P., 1982. An inverse approach to signal correlation. J. Geophys. Res., 87:4807-4818.

Moore, T. C., Jr., and Romine, K., 1981. In search of biostratigraphic resolution. Soc. Econ. Paleontol. Mineral. Spec. Publ., 32:317-334.

Pisias, N. G., Martinson, D. G., Moore, T. C., Jr., Shackleton, N. J., Prell, W., et al., 1984. High resolution stratigraphic correlation of

Table 3. Stratigraphic resolution for Leg 85 drill sites (ranges of correlation based on radiolarian, diatom, and carbonate stratigraphies).

\begin{tabular}{llcc}
\hline & & $\begin{array}{c}\text { Mean } \\
\text { (m at } \\
\text { Site 573) }\end{array}$ & $\begin{array}{c}\text { Standard } \\
\text { deviation } \\
\text { (m at } \\
\text { Site 573) }\end{array}$ \\
\hline 572 & (all) & 3.4 & 2.9 \\
572 & (Pliocene only) & 3.3 & 1.0 \\
574 & (all) & 3.8 & 2.5 \\
575 & (all) & 9.8 & .7 .1 \\
575 & (below 160 m) & 3.6 & 1.2 \\
\hline
\end{tabular}

benthic oxygen isotope records spanning the last 300,000 years. Mar. Geol., 56:119-136.

Shaw, A. B., 1964. Time in Stratigraphy: New York (McGraw-Hill).

Date of Initial Receipt: 6 March 1984

Date of Acceptance: 19 June 1984

Table 4. Resolution of biodatums (general statistics for depth ranges over which biodatums were determined).

\begin{tabular}{llcc}
\hline & Fossil & $\begin{array}{c}\text { Mean } \\
(\mathrm{m})\end{array}$ & $\begin{array}{c}\text { Standard } \\
\text { deviation } \\
(\mathrm{m})\end{array}$ \\
\hline 572 & Diatoms & 2.8 & 0.7 \\
572 & Radiolarians & 6.0 & 2.9 \\
573 & Diatoms & 4.0 & 2.7 \\
573 & Radiolarians & 6.2 & 2.2 \\
574 & Diatoms & 4.0 & 2.5 \\
574 & Radiolarians & 5.3 & 3.0 \\
575 & Diatoms & 2.7 & 2.2 \\
575 & Radiolarians & 3.6 & 2.4 \\
\hline
\end{tabular}


N. G. PISIAS, J. A. BARRON, C. A. NIGRINI, D. A. DUNN

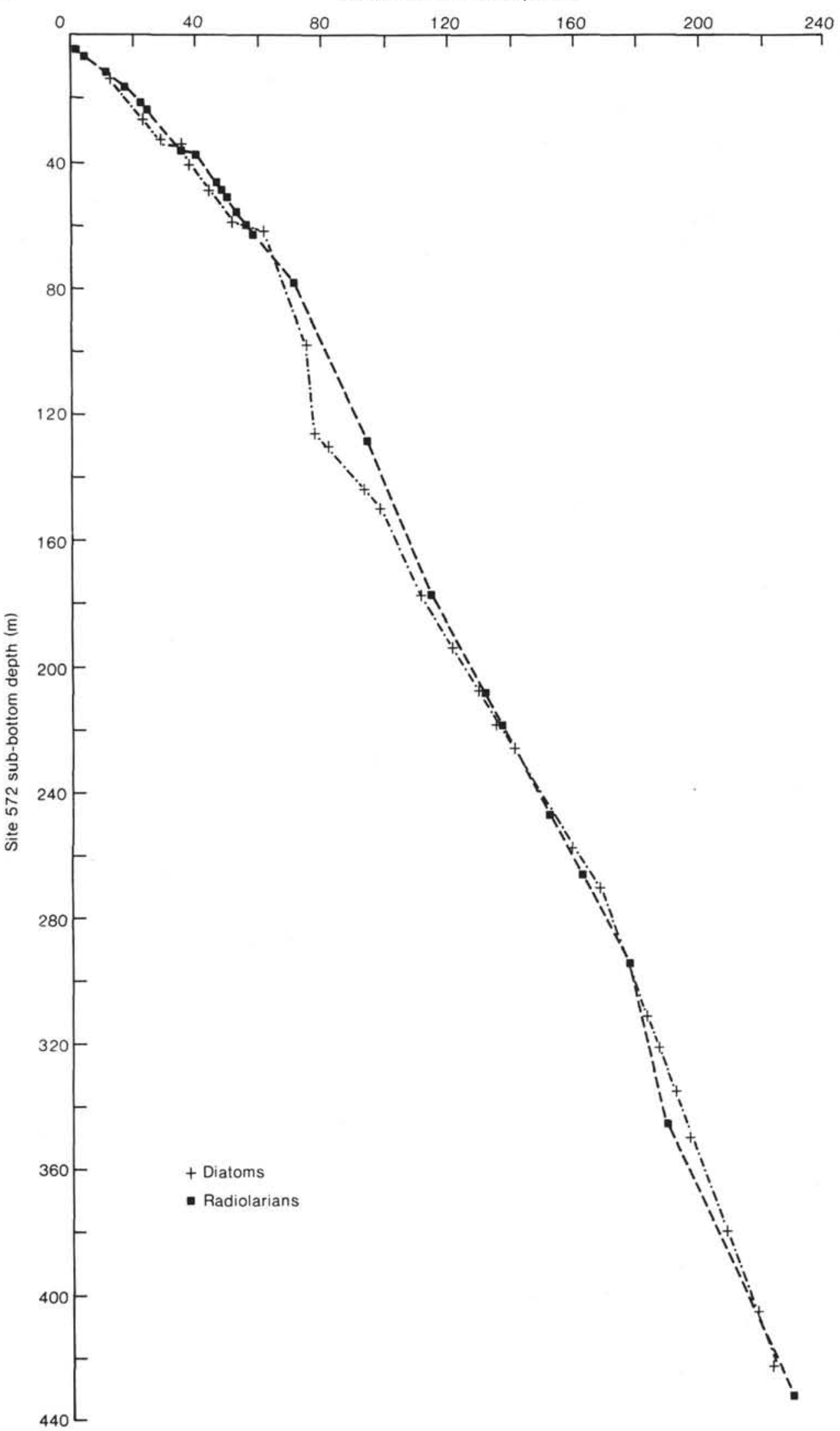

Figure 4. Lines of correlation determined from both biodatums and carbonate records. A. Site 572 versus Site 573. B. Site 574 versus Site 573. C. Site 575 versus Site 573. 

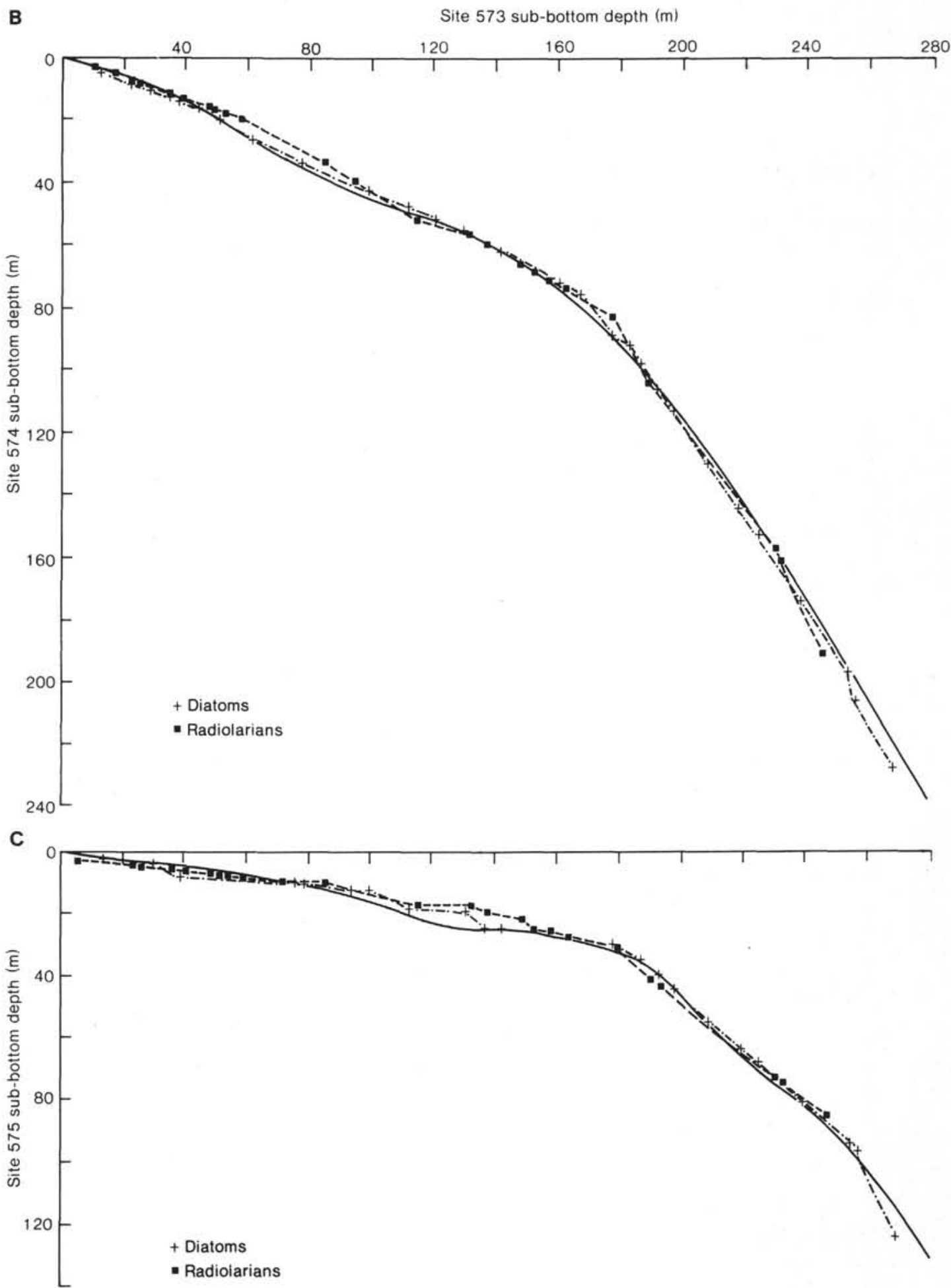

Figure 4. (Continued). 
N. G. PISIAS, J. A. BARRON, C. A. NIGRINI, D. A. DUNN

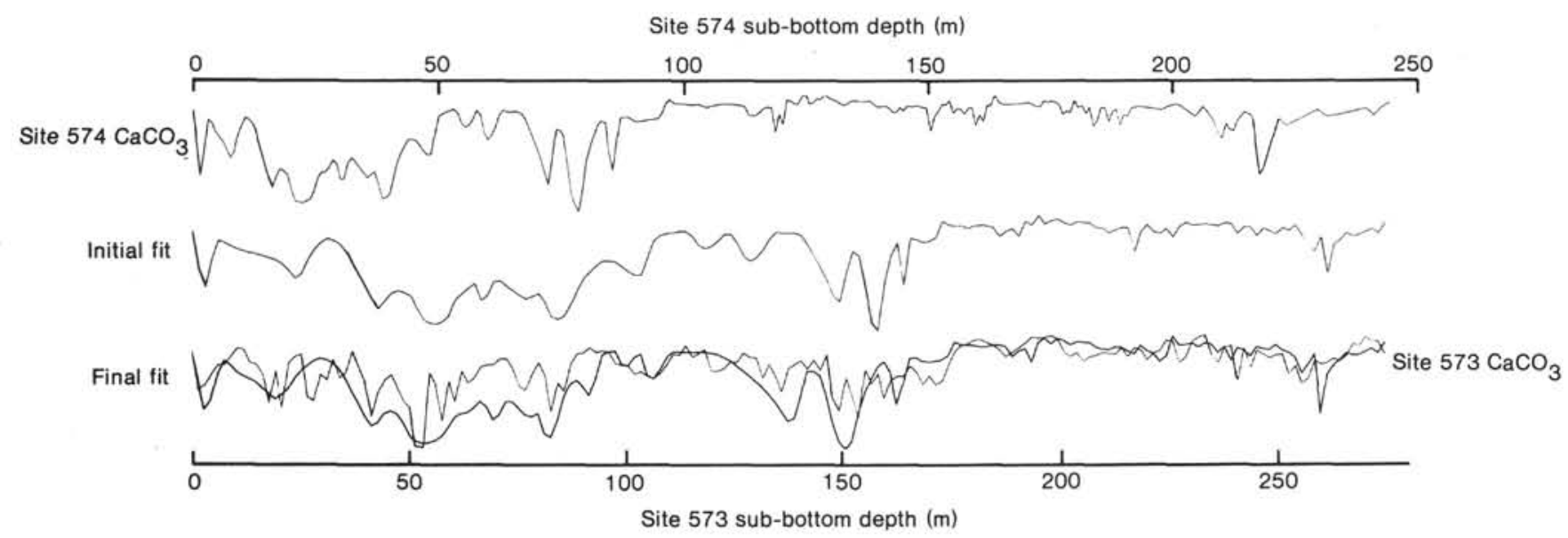

Figure 5. Carbonate plots comparing Sites 574 and 573. 